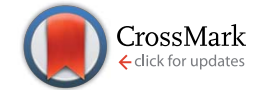

Cite this: RSC Adv., 2017, 7, 4904
Received 30th November 2016 Accepted 5th January 2017

DOI: 10.1039/c6ra27571k

www.rsc.org/advances

\section{The solvothermal synthesis of $\gamma$-AlOOH nanoflakes and their compression behaviors under high pressures}

\author{
Xudong Zhou, ${ }^{a}$ Jian Zhang, $\dagger^{\star a}$ Yanmei Ma, $\dagger^{\star b}$ Hui Tian, ${ }^{a}$ Yue Wang, ${ }^{a}$ Yingai $\mathrm{Li}{ }^{a}$ \\ Lina Jiang ${ }^{a}$ and Qiliang Cui ${ }^{a}$
}

Boehmite, $\gamma-\mathrm{AlOOH}$, has attracted increasing research enthusiasm due to its scientific and technological importance. The compression behaviors of $\gamma-\mathrm{AlOOH}$ are of vital relevance to the insight into geophysical and geochemical processes in Earth's mantle. In this work, free-standing $\gamma-\mathrm{AlOOH}$ nanoflakes with high purity and crystallinity have been synthesized via a template-free, one-step solvothermal alcoholysis strategy. The nanoflakes have high aspect ratio, with the lateral size ranging from 100 to $300 \mathrm{~nm}$, and the thickness ranging from 20 to $45 \mathrm{~nm}$. The band gap of the obtained $\gamma$-AlOOH nanoflakes is estimated to be $3.35 \mathrm{eV}$ through UV-vis absorption spectra recorded at room temperature. A broad PL emission band can be observed in the 400 to $700 \mathrm{~nm}$ spectrum range, with the maximum at $480 \mathrm{~nm}$. The compression behaviors of $\gamma-\mathrm{AlOOH}$ nanoflakes have been investigated by in situ high pressure synchrotron radiation angle dispersive X-ray diffraction up to $54.9 \mathrm{GPa}$ and Raman scattering spectroscopy up to $22.4 \mathrm{GPa}$ at room temperature. The compression is nearly linear and anisotropic, with $b$ axis more compressible than $a$ and $c$ axes. The zero-pressure bulk modulus of $\gamma$-AlOOH nanoflakes is estimated to be $135.2 \mathrm{GPa}$, higher than the theoretical value of its bulk counterpart. The frequencies of the observed $\mathrm{Al}-\mathrm{O}$ related Raman modes increase monotonously in a linear manner with increasing pressure. The mode Grüneisen coefficients are estimated, which may provide important information about the anharmonicity in the bonding nature of $\gamma-\mathrm{AlOOH}$.

\section{Introduction}

Nanoscale materials with tailored morphologies may exhibit novel physical and chemical properties dependent on their dimensions, sizes, shapes, and exposed facets. ${ }^{\mathbf{1 , 2}}$ The subsequent utilization of nanocrystals with tailored geometries as building blocks for the fabrication of nanodevices offers the opportunity to control and manipulate the properties and applications of materials as desired. Hence, materials prepared via 'nanoshape engineering' have become one of the most promising and challenging issues of nowadays science and technology. Among these materials are nanoscale $\gamma$-AlOOH, boehmite, which have attracted increasing research enthusiasm on the synthetic methodology and potential applications recently. Various $\gamma$-AlOOH nanostructures with controllable geometries, such as 0D nanoparticles, 1D nanorods and nanowires, 2D nanosheets and nanoflakes, have been prepared and showed versatile potential applications in the fields of biomedicine, environmental chemistry, catalyst, sorbent,

\footnotetext{
${ }^{a}$ State Key Laboratory of Superhard Materials, Jilin University, Changchun 130012, China.E-mail: zhang jian@jlu.edu.cn; ymma@jlu.edu.cn

${ }^{b}$ College of Physics, Jilin University, Changchun 130012, China

$\dagger$ Jian Zhang and Yanmei Ma contributed equally to this work.
}

ceramic, and optical nanodevices. For instance, a library of $\gamma$-AlOOH nanoparticles including nanorods, nanoplates and nano-polyhedra with defined shape, crystallinity, and hydroxyl content, have been designed and synthesized, and these physicochemical properties have been demonstrated to play a key role in the ability of $\gamma-\mathrm{AlOOH}$ to activate superior immune responses. ${ }^{3}$ Novel 2D $\gamma$-AlOOH porous nanosheets have been prepared solvothermally, which may be converted to $\mathrm{Al}_{2} \mathrm{O}_{3}$ nanosheets by thermal decomposition. ${ }^{4}$ The obtained sheet-like $\mathrm{Al}_{2} \mathrm{O}_{3}$ may be used as a nanocatalyst support. The flowerlike $\gamma$-AlOOH nanoarchitectures, with three hierarchies of self organization, i.e., single-crystalline nanorods, nanostrips, and bundles, have been sequentially synthesized, which exhibited high BET area and excellent porous properties. ${ }^{5}$ Typically, hydrothermal and/or solvothermal procedures are involved in the synthetic strategies of these $\gamma$-AlOOH nanomaterials. Through delicate control of the reaction parameters, such as the $\mathrm{pH}$ value, the temperature, the time of processing, and the selection of precursors, solvents, and surfactants, etc., tuning of the dimension, size and shape of the target $\gamma$-AlOOH nanomaterials as desired may be achieved..$^{6-16}$

In addition to its technological importance, $\gamma$-AlOOH is also of considerable relevance in high pressure studies. As a mineral with intrastructural hydroxyl groups, the structure and stability 
of boehmite under high pressures is not only relevant for the investigation of geophysical and geochemical processes in the Earth's crust and the upper mantle, but also for the preparation of new materials with specific properties via high pressure/high temperature techniques. $\gamma$-AlOOH crystallizes in the orthorhombic dipyramidal structure, which may be described in space group $\mathrm{Cmcm}$, or $C m c 2_{1}$ and $P 2_{1} / c{ }^{17-20}$ The difference among them lies in the position of $\mathrm{H}$ atoms only, with the Al-O frame being essentially the same. The unit cell is constituted by distorted $\mathrm{AlO}_{3}(\mathrm{OH})_{3}$ octahedra. The $\mathrm{AlO}_{3}(\mathrm{OH})_{3}$ octahedra contain two types of oxygen atoms: $\mathrm{O}_{\mathrm{I}}$ has four Al neighbors, $\mathrm{O}_{\text {II }}$ has but two $\mathrm{Al}$ neighbors and forms covalent bond with the $\mathrm{H}$ atom. The $\mathrm{AlO}_{3}(\mathrm{OH})_{3}$ octahedra interconnect to form 2D layers perpendicular to the $b$ axis. Each layer possesses hydroxyl $\mathrm{O}_{\mathrm{II}}-\mathrm{H}$ groups on both sides and a zigzag chain of hydrogen bonds of the $\mathrm{O}_{\mathrm{II}}-\mathrm{H} \cdots \mathrm{O}_{\text {II }}$ type is formed between the layers. The plane of the hydrogen bond chain is parallel to the $b-c$ crystallographic plane. $\gamma$-AlOOH is polymorphous with $\alpha$-AlOOH (diaspore) and $\delta$-AlOOH (a metastable AlOOH phase synthesized under high pressures and high temperatures and quenched to ambient conditions). ${ }^{21-23} \alpha-\mathrm{AlOOH}$ is considered as a pertinent model system for high pressure studies of hydrogen bonds. The high pressure behaviors of $\alpha$-AlOOH have been the matter of intensive experimental and theoretical studies. ${ }^{24-30}$ It has been found that $\alpha$-AlOOH remains stable up to at least $15 \mathrm{GPa}$ and $1123 \mathrm{~K}$. The compression of the crystal structure of $\alpha$-AlOOH proceeds mainly by bond shortening, and particularly by compression of the hydrogen bond. The hydrogen bond in $\alpha$-AlOOH becomes significantly more symmetric with increasing pressure, but a complete symmetrization has not been reached in the investigated pressure range and does not seem likely to occur even at higher pressures. Initial researches on $\delta$-AlOOH indicated its phase stability under a wide temperature range of less than $2300 \mathrm{~K}$ and pressure range from 18 to $134 \mathrm{GPa}^{21-23,31}$ However, recent investigations implied that an order-disorder phase transition would occur in $\delta$-AlOOH between 6.1 and $8.2 \mathrm{GPa}$, concerning the situation of $\mathrm{H}$ distribution. ${ }^{32,33}$ The structural, mechanical and spectroscopic properties of $\gamma$-AlOOH under high pressures have been investigated by means of firstprinciple density functional theory (DFT). ${ }^{34}$ Isostatic structural compression via the DFT method gives the bulk modulus of 97 GPa in the athermal limit, for which mainly a strong shortening mechanism of interlayer hydrogen bonds is responsible. In contrast to the continuous research enthusiasm on the $\alpha$ - and $\delta$ polymorphs of AlOOH, there are scarcely detailed experimental data for $\gamma$-AlOOH under high pressure conditions.

In this work, the compression behaviors of $\gamma$-AlOOH under high pressure conditions are reported. The samples under study are high purity, free-standing $\gamma$-AlOOH nanoflakes synthesized via a facile, template-free, one-step solvothermal alcoholysis method. The structural stabilities and mechanical properties have been investigated by in situ synchrotron radiation angledispersive X-ray diffraction (ADXRD) and Raman scattering techniques. These experimental results will help to further investigate the undisclosed intrinsic physical properties of $\gamma$-AlOOH nanomaterials. We expect that this study will not only provide valuable high pressure experimental information of
$\gamma$-AlOOH, but also offer the opportunity for a better understanding of the hydrogen bond related compression behaviors of other layered compounds with intrastructural hydrogen bond, especially at the nanoscale.

\section{Experimental section}

\subsection{Synthesis of $\gamma$-AlOOH nanoflakes}

All chemicals were analytical grade reagents and were used without further purification. For the synthesis of $\gamma$-AlOOH nanoflakes, powders of $0.2 \mathrm{~g}$ anhydrous aluminum chloride $\left(\mathrm{AlCl}_{3}, \mathrm{AR}\right.$, Sinopharm Chemical Reagent Co., Ltd, China) were added into $15 \mathrm{~mL}$ methanol. The mixture was agitated vigorously by a magnetic stirrer for 20 minutes and a clear solution was formed. The resulting solution was transferred to a Teflon lined stainless steel autoclave, which was tightly sealed and heated at a rate of $2{ }^{\circ} \mathrm{C} \mathrm{min}-1$ in an electric oven to $350{ }^{\circ} \mathrm{C}$ and maintained for 3 hours. When the autoclave was cooled naturally to ambient temperature, the products were collected and washed with acetone several times to remove the residual reagents, and then dried at $60^{\circ} \mathrm{C}$ for 12 hours. During the whole synthesizing process, there was no need for any shape-directing additives or regulating the $\mathrm{pH}$ value of the reaction environment.

\subsection{Characterization}

Structural analysis of the products was carried out by powder X-ray diffractometry on a Shimadzu XRD-6000 diffractometer working with $\mathrm{Cu} \mathrm{K} \alpha$ radiation $(\lambda=0.154178 \mathrm{~nm})$. The SEM images of the samples were taken on a HITACHI S4800 microscope working at $25.0 \mathrm{kV}$, which is equipped with an energy dispersive spectrometer (EDS). The TEM micrographs of the samples, as well as the selected area electron diffraction (SAED) patterns, were obtained via a JEM-2200FS transmission electron microscope using an accelerating voltage of $200 \mathrm{kV}$. The ultraviolet-visible (UV-vis) absorption spectra of the synthesized product were taken on a Shimadzu UV-3150 spectrometer, and the photoluminescence (PL) measurement was conducted under $250 \mathrm{~nm}$ UV fluorescent light excitation by a Shimadzu RF5301 PC spectrophotometer at room temperature.

\subsection{High pressure studies}

High pressure experiments were conducted by using a symmetric diamond anvil cell (DAC) furnished with $400 \mu \mathrm{m}$ culet diamonds. The T301 stainless steel gasket was preindented by the diamonds to an initial thickness of about $50 \mu \mathrm{m}$ and then a center hole of $90 \mu \mathrm{m}$ in diameter was drilled as the sample chamber. The samples with the liquid quasihydrostatic pressure-transmitting medium (silicone oil) were loaded into the sample chamber along with a tiny ruby chip for pressure measurements. The pressure within the sample chamber is increased in a stepwise manner, with intervals of 1-2 GPa. The pressure was determined from the frequency shift of the ruby R1 fluorescence line. The R1 and R2 lines of the ruby fluorescence signal remained well separated throughout the experiments, indicating that a good quasi-hydrostatic condition 
was maintained even when the pressure medium was solidified. The angle-dispersive XRD experiments were carried out with a wavelength of $0.6199 \AA$ at the $4 \mathrm{~W} 2$ High-Pressure Station of Beijing Synchrotron Radiation Facility (BSRF). The synchrotron X-ray beam was focused to a spot of about $29 \times 15 \mu \mathrm{m}^{2}$ in size. The average acquisition time was $300 \mathrm{~s}$. The sample-detector distance and geometric parameters were calibrated using a $\mathrm{CeO}_{2}$ standard from NIST. The Bragg diffraction rings were recorded by using a Mar345 CCD detector and the one dimensional diffraction profiles of intensity as a function of $2 \theta$ were obtained by integration of the observed two dimensional patterns with the Fit2D software. ${ }^{35}$ Lattice parameters of the samples were obtained with Rietveld refinements of the powder XRD patterns performed using GSAS program packages. ${ }^{36}$ In situ high pressure Raman scattering spectra were collected at room temperature by using an Acton SpectraPro 500i spectrometer with $1800 \mathrm{~g} \mathrm{~mm}^{-1}$ holographic grating and a liquid nitrogen cooled CCD detector (Princeton Instruments, $1340 \times 100$ ). A solid-state diode-pumped $\mathrm{Nd}$ : vanadate laser (Coherent Inc.) with the output wavelength of $532 \mathrm{~nm}$ was employed as the excitation source. Pressure-induced shifts of Raman scattering bands were analyzed by fitting the spectra to Lorentzian functions to determine the line-shape parameters.

\section{Results and discussion}

The typical XRD patterns of the prepared samples are shown in Fig. 1(a). All the detectable peaks can be unambiguously assigned by their peak positions to $\gamma$-AlOOH, as compared with
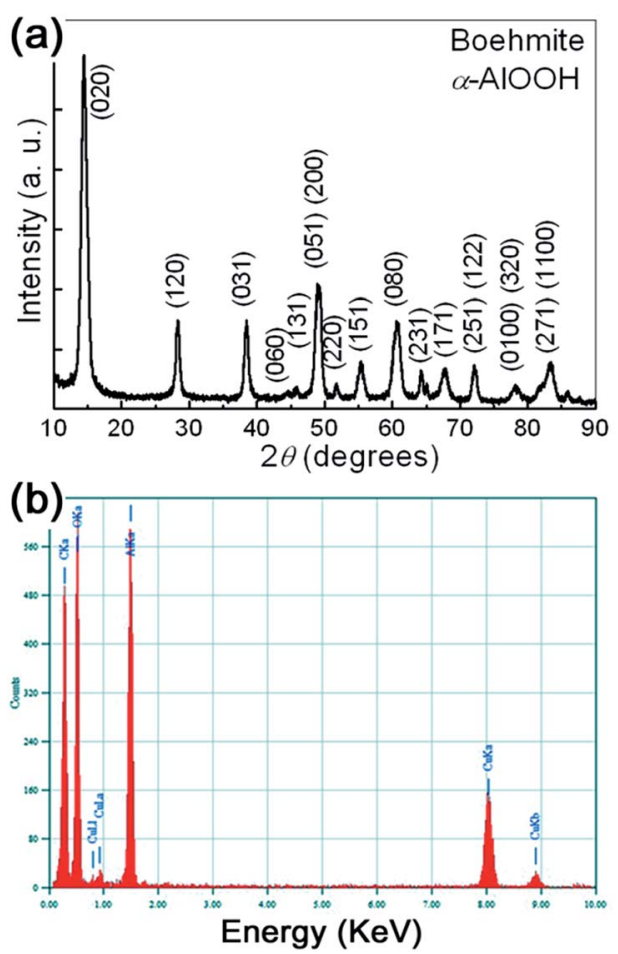

Fig. 1 (a) The typical XRD pattern of the prepared samples. (b) The typical EDS spectra of the prepared samples. the standard diffraction patterns (PDF no. 21-1307). No additional peaks belonging to other $\mathrm{AlOOH}$ phases or impurities can be observed, indicating the high purity of the obtained samples. The sharp peaks also indicate that the prepared samples have perfect crystallinity. The lattice parameters of the synthesized $\gamma$ AlOOH samples are determined by Rietveld refinements of the powder XRD patterns. The results indicate that the obtained $\gamma$ AlOOH samples can be indexed to an orthorhombic cell with lattice parameters $a=0.369 \mathrm{~nm}, b=1.225 \mathrm{~nm}, c=0.286 \mathrm{~nm}$.

In addition to XRD results, the chemical composition of the prepared samples was further checked by energy dispersive Xray analysis (EDS). The typical EDS spectra is shown in Fig. 1(b). It can be seen that the prepared $\gamma$-AlOOH sample contains mainly $\mathrm{O}$ and $\mathrm{Al}$ elements. $\mathrm{C}$ and $\mathrm{Cu}$ elements coming from the supporting mesh are also detected. In a wide spectrum range, no signals for other elements can be observed. Therefore, both XRD and EDS analyses show that pure orthorhombic phase $\gamma$-AlOOH with high crystallinity is successfully synthesized by the present one-step synthetic strategy.

To provide further insight into the structures of the prepared $\gamma$-AlOOH samples, the morphology of the prepared $\gamma$-AlOOH samples are observed by SEM and TEM techniques. The typical panoramic SEM image of the sample, as shown in Fig. 2(a), reveals that the sample consists of large quantities of small,
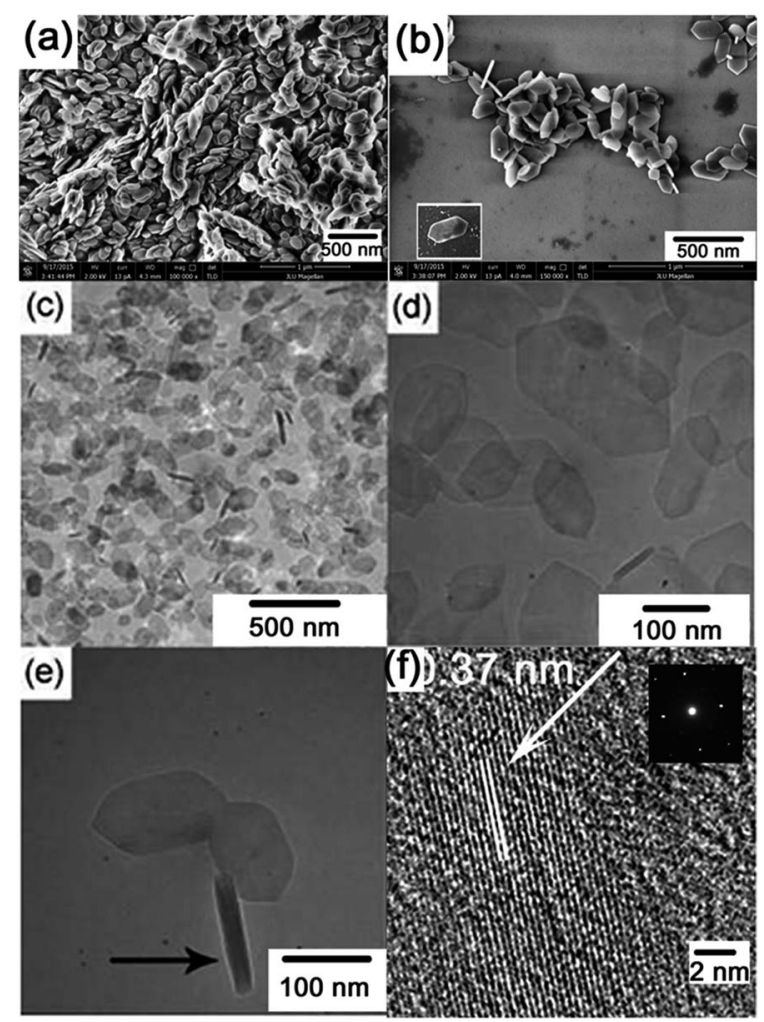

Fig. 2 (a) The typical panoramic SEM image of the prepared sample. (b) The SEM image of the sparsely dispersed samples. The inset shows an isolated nanoflake with well-defined shape. (c) and (d) show the overall TEM images at low and high magnification, respectively, of the prepared samples. (e) shows several isolated nanoflakes with different orientations. (f) The HRTEM image and the corresponding SAED pattern of an individual nanoflake. 
thin flakes. The flakes have a quasi-hexagonal shape. From the SEM image of the sparsely dispersed samples (Fig. 2(b)) it can be seen that the nanoflakes have high aspect ratio. The lateral size of them ranges from 100 to $300 \mathrm{~nm}$, and the thickness ranges from 20 to $45 \mathrm{~nm}$. Most of the nanoflakes have well-defined outlines, as shown in the inset of Fig. 2(b). Fig. 2(c) shows the overall TEM image at low magnification of the prepared $\gamma$ AlOOH samples. It confirms that the sample comprises large numbers of nanometer scaled flakes. From the high magnification TEM image shown in Fig. 2(d), it can be validated that the quasi-hexagonal nanoflakes are fairly thin, as the overlapping of them can be easily evidenced. In Fig. 2(e) are shown several isolated nanoflakes with different orientations. The one with an orientation perpendicular to others shows clearly its thickness of about $25 \mathrm{~nm}$. The HRTEM image and the corresponding selected area electron diffraction (SAED) pattern of an individual nanoflake, shown in Fig. 2(f), reveal that the nanoflakes are highly single crystalline in nature. The distance between fringes is measured to be about $0.37 \mathrm{~nm}$, very close to the (100) interplanar $d$-spacing of the orthorhombic $\gamma$-AlOOH phase, which is in good accordance with the XRD studies.

The typical UV-vis absorption spectrum of the prepared sample is shown in Fig. 3(a). An absorption edge can be observed in the $250-500 \mathrm{~nm}$ range, which may be reasonably ascribed to $\mathrm{O}^{2-} \rightarrow \mathrm{Al}^{3+}$ charge transfer transitions. ${ }^{37}$ The broadening of the absorption edge is indicated to be related with the dispersed particle sizes. In order to determine the optical bandgap of the sample, the well known Kubelka-Munk transformation is used as follows:
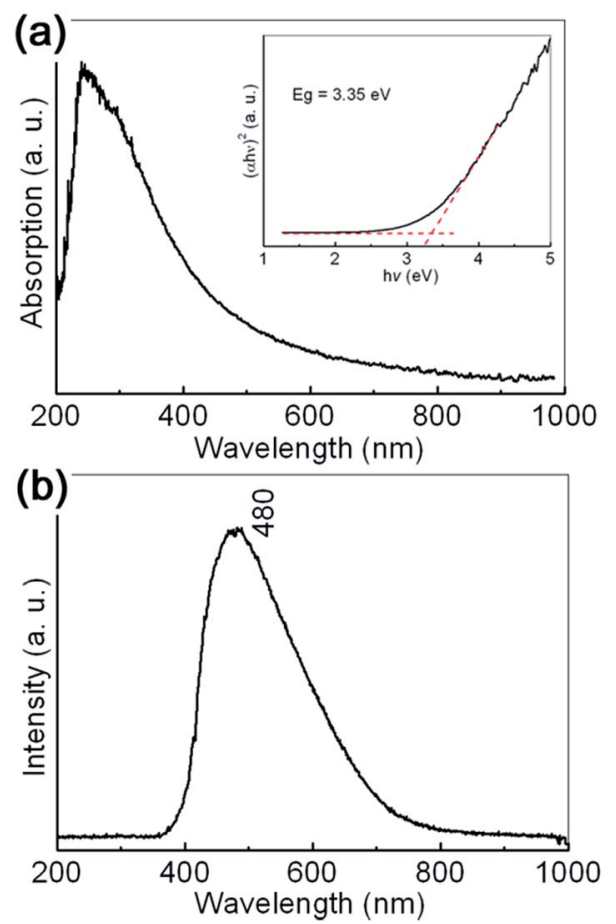

Fig. 3 (a) The typical UV-vis absorption spectrum of $\gamma-\mathrm{AlOOH}$ nanoflakes. The inset shows the plot of $(\alpha h \nu)^{2}$ versus $h \nu$ according to Kubelka-Munk transformations. (b) The typical PL spectrum of $\gamma$ $\mathrm{AlOOH}$ nanoflakes.

$$
(\alpha h \nu)^{2}=A\left(h \nu-E_{\mathrm{g}}\right)
$$

where $\alpha$ is the absorption coefficient, $A$ is a constant, $h$ is the Planck's constant, $\nu$ is the photon frequency, and $E_{\mathrm{g}}$ is the optical bandgap. Since $E_{\mathrm{g}}=h \nu$, when $(\alpha h \nu)^{2}=0$, an extrapolation of the linear region of the plot of $(\alpha h \nu)^{2}$ versus photon energy $(h \nu)$ on the transverse axis corresponds to the value of the optical bandgap e.g. as shown in the inset of Fig. 3(a), by extrapolation of the linear region, the average optical bandgap of the obtained $\gamma$-AlOOH nanoflakes can be estimated to be about $3.35 \mathrm{eV}$. The computational calculations of the electronic band structure of $\gamma-\mathrm{AlOOH}$, obtained at the density functional theory (DFT) level, indicated that $\gamma$-AlOOH has a direct energy bandgap of $4.51 \mathrm{eV}$. However, the experimental values were sensitive to the shape and size of the samples. The bandgap of $\gamma$-AlOOH nanomaterials varied from $3.87 \mathrm{eV}$ for nanowires with diameter of $30-40 \mathrm{~nm}$, to 4.54 and $4.67 \mathrm{eV}$ for nanoparticles with diameter of several hundreds of nanometers. ${ }^{37}$ It seemed that smaller sizes favored smaller bandgaps. The samples in this study are nanoflakes with the thickness of 20-45 nm, thus a bandgap a little smaller than that of the $30-40 \mathrm{~nm}$ nanowires is reasonable.

The typical room temperature PL spectrum of the synthesized $\gamma$-AlOOH nanoflakes is shown in Fig. 3(b). A broad, asymmetric PL emission band can be clearly observed in the 400 to $700 \mathrm{~nm}$ range, with the maximum at about $480 \mathrm{~nm}(2.58 \mathrm{eV})$. Generally speaking, the optical properties of $\gamma$-AlOOH nanomaterials are not fully understood. However, it is believed that they are strongly dependent on the morphological features, such as size and shape. For example, the center position of the main peak observed for the ultrathin $\gamma$-AlOOH nanobelts with an average width of $10 \mathrm{~nm}, 298 \mathrm{~nm}(4.16 \mathrm{eV})$, exhibits an obvious blue shift to that observed for the $\gamma$-AlOOH nanowhiskers with an average width of $25 \mathrm{~nm}, 360 \mathrm{~nm}(3.44 \mathrm{eV})$. At the same time, structural defects, such as impurities and color centers $\left(\mathrm{F}\right.$ and $\mathrm{F}^{+}$), may play an important role in the charge transfer and luminescent transitions. ${ }^{37-39}$

It is well-accepted that applying high pressure is an effective approach to study the structural stability and elastic properties of bulk/nanomaterials. The as-prepared $\gamma$-AlOOH nanoflakes are investigated by using in situ high pressure angle dispersive synchrotron radiation X-ray diffraction (ADXRD) techniques. Representative XRD patterns under high pressures up to 54.9 GPa are shown in Fig. 4(a) and the pressure dependent $d$-spacings of the lattice planes, as well as the unit-cell parameters, are shown in Fig. 4(b) and (c). The diffraction peaks are denoted with Miller indices and can be indexed to the orthorhombic cell of $\gamma$-AlOOH. During the entire compression processes, all diffraction peaks shift to higher angles with increasing pressure and no extra peaks appear in the XRD patterns. The corresponding $d$-spacings decrease approximately in a linear manner with increasing pressure. A similar variation of the unit-cell parameters is also observed. However, by taking a closer look at the lattice parameters, a strong anisotropy of the linear compressibilities of $\gamma-\mathrm{AlOOH}$ is obvious, with the $b$ axis being much more compressible than the $a$ and $c$ axes. It is reasonable, since in boehmite, the $\mathrm{Al}-\mathrm{O}$ framework forms two- 

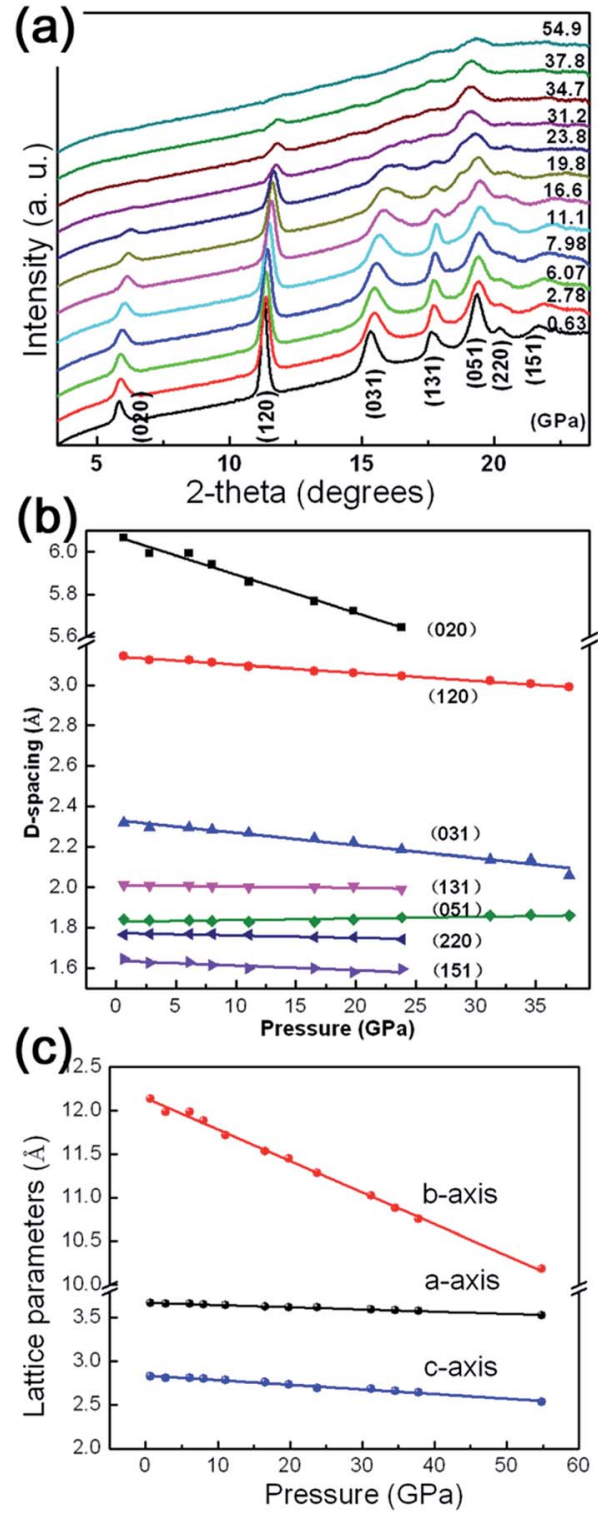

Fig. 4 (a) Representative XRD patterns under high pressures of $\gamma$ $\mathrm{AlOOH}$ nanoflakes. (b) The pressure dependent $d$-spacings of $\gamma$ $\mathrm{AlOOH}$ nanoflakes. (c) The variation of the lattice paremeters of $\gamma$ $\mathrm{AlOOH}$ nanoflakes under high pressures.

dimensional layers connected via hydrogen bonds. Therefore, its structure is strongly anisotropic and the most compressible along the direction of the hydrogen bonds perpendicular to the $a-c$ plane. Taking the high-pressure XRD results into account, it is suggested that there are no phase transitions in the pressure ranges we achieved in this study. To our knowledge, this is the first time that the effects of pressure on $\gamma$-AlOOH have been studied experimentally. Our results are in accordance with the theoretical studies that $\gamma$-AlOOH is stable under high pressures. $^{34}$

The information on the mechanical properties of $\gamma$-AlOOH can be obtained through investigation of the dependence of the structure upon compression. Variation of unit-cell volume with pressure is plotted in Fig. 5(a). It can be seen that the unit-cell
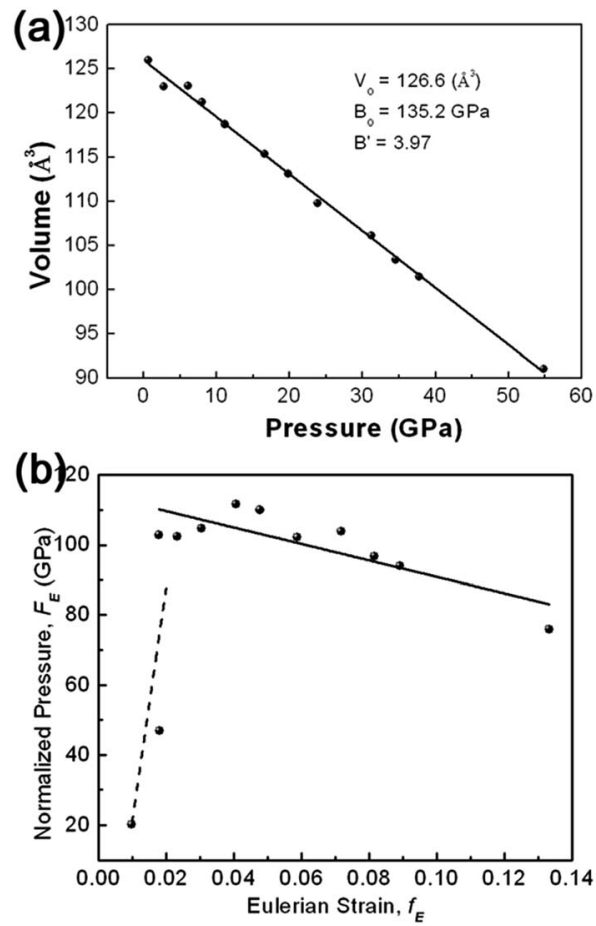

Fig. 5 (a) The unit-cell volume as a function of pressure, which is fitted to the third order Birch-Murnaghan equation of state. (b) The volumepressure data displayed as a plot of the normalized pressure $F_{\mathrm{E}}$ against the Eulerian strain $f_{\mathrm{E}}$. The solid line represents the linear fit of data above $6.07 \mathrm{GPa}$, while the dashed line is only a guide to the eyes.

volume of $\gamma$-AlOOH decrease linearly, at least as a first order approximation, with increasing pressure. Fitting the pressurevolume data to the third-order Birch-Murnaghan (BM) equation of state (EOS) yields the bulk modulus of $B_{0}=135.2 \mathrm{GPa}$ with $B^{\prime}{ }_{0}$ $=3.9$. Owing to the lack of other experimental data for $\gamma-\mathrm{AlOOH}$ in the literature, no direct comparison can be performed. However, theoretical values are available. The structural, mechanical and spectroscopic properties of $\gamma$-AlOOH have been investigated by means of first-principle density functional theory (DFT) and semiempirical density functional based tight binding (DFTB) methods. ${ }^{34}$ Isostatic structural compression was performed and the bulk modulus spreading in the range of 82-97 GPa was obtained, exhibiting an enhanced compressibility compared with our experimental results.

In order to obtain a direct indication of the compression behavior and of the quality of the EOS fitting, the compression curve shown as plot of unit cell volume against pressure $(P-V$ plot) is transformed into plot of finite strain $f_{\mathrm{E}}$ against normalized stress $F_{\mathrm{E}}\left(F_{\mathrm{E}}-f_{\mathrm{E}}\right.$ plot), as illustrated in Fig. 5(b). The $F_{\mathrm{E}}-f_{\mathrm{E}}$ plot is useful to visualize slight anomalies of equation of state that is difficult to be found in the $P-V$ plot. For BirchMurnaghan equation of state, $f_{\mathrm{E}}$ and $F_{\mathrm{E}}$ are defined as follows:

$$
\begin{gathered}
f_{\mathrm{E}}=\left[\left(V_{0} / V\right)^{2 / 3}-1\right] / 2 \\
F_{\mathrm{E}}=P /\left[3 f_{\mathrm{E}}\left(1+2 f_{\mathrm{E}}\right)^{5 / 2}\right]
\end{gathered}
$$


In the $F_{\mathrm{E}}-f_{\mathrm{E}}$ plot, the slope is equal to $3 B_{0}\left(B_{0}^{\prime}-4\right) / 2$, and the intercept on the vertical $F_{\mathrm{E}}$ axis is the value of $B_{0}$. It can be seen from Fig. 5(b) that all the data points except the first two (at low pressures) reside roughly in a line with a slope slightly lower than 0 . A linear fitting of the $F_{\mathrm{E}}-f_{\mathrm{E}}$ data yields $B_{0}=114.4 \mathrm{GPa}$ and $B^{\prime}{ }_{0}=2.6$, consistent qualitatively with the fitting of the $P-V$ data to the third-order BM-EOS.

Concerning the experimental studies on the mechanical properties of materials under high pressures, several factors should be taken into accounts, such as hydrostaticity of pressure and the effects of size and shape, which would affect the compressibility. Systematical studies on the compressibility of nanocrystalline materials showed that the bulk modulus is significantly enhanced when measured under nonhydrostatic conditions. ${ }^{\mathbf{4 0 , 4 1}}$ In this study, hydrostatic stress is achieved by immersing the sample in a pressurized fluid medium (silicone oil). This type of medium is able to maintain a hydrostatic/ quasihydrostatic state up to $15 \mathrm{GPa}^{42}$ Thus the effect of the pressure-induced non-hydrostaticity in the sample chamber could be a possible reason for the enhanced bulk modulus. Pressure profoundly alters matters and materials, especially when they are at the nanoscale. The morphological features of nanomaterials, such as size and shape, play a very important role on their compression and structural behaviors under pressure. Generally speaking, the decrease in the grain size of nanocrystalline materials brings in turn an increase in the bulk modulus, as compared with their bulk counterparts. ${ }^{\mathbf{4 3 4 4}}$ This phenomena might be related with the higher surface energy contribution, more grain boundaries and fewer dislocations, of nanocrystalline materials. In our case, the thickness of the $\gamma$ $\mathrm{AlOOH}$ nanoflakes under study ranges from 20 to $45 \mathrm{~nm}$, implying that they can exhibit an enhanced strength against compression, i.e., reduced compressibility.

In order to obtain more detailed insight into the question of the high anisotropy of the compressibility, the dependences of the intralayer $\mathrm{Al}-\mathrm{O}$ and interlayer hydrogen bonds on the volume compression have been studied by using Raman scattering spectroscopy. From the viewpoint of group theory, based on the decomposition of the reducible representation built on the basis of the Cartesian coordinates of the unit cell atoms, the symmetry of the normal modes at the Brillouin zone center $(\Gamma$ point) of $\gamma$-AlOOH, in the space group of either $\mathrm{Cmcm}$ or $\mathrm{Cmc2}_{1}$, may be assigned as the following:

$$
\begin{gathered}
\Gamma^{C m c m}=4 \mathrm{~B}_{1 \mathrm{u}}+4 \mathrm{~B}_{2 \mathrm{u}}+4 \mathrm{~B}_{3 \mathrm{u}}+4 \mathrm{~B}_{1 \mathrm{~g}}+4 \mathrm{~B}_{2 \mathrm{~g}}+4 \mathrm{~A}_{\mathrm{g}} \\
\Gamma^{C m c 2_{1}}=8 \mathrm{~A}_{1}+4 \mathrm{~A}_{2}+8 \mathrm{~B}_{1}+4 \mathrm{~B}_{2}
\end{gathered}
$$

For the space group $\mathrm{Cmcm}$, the Raman active modes are $4 \mathrm{~B}_{1 \mathrm{~g}}$ $+4 \mathrm{~B}_{2 \mathrm{~g}}+4 \mathrm{~A}_{\mathrm{g}}$. For the space group $C m c 2_{1}$, the Raman active modes are $7 \mathrm{~A}_{1}+4 \mathrm{~A}_{2}+7 \mathrm{~B}_{1}+3 \mathrm{~B}_{2}$. The selected Raman scattering patterns of $\gamma$-AlOOH nanoflakes at various pressures are shown in Fig. 6(a) for the modes related with the Al-O bonds (200-800 $\mathrm{cm}^{-1}$ ), and in Fig. 6(b) for the modes related with the hydrogen bonds (2800-3400 $\left.\mathrm{cm}^{-1}\right)$. In the low frequency region, five features may be distinguished with their Raman shifts at about $363,456,499,620$, and $685 \mathrm{~cm}^{-1}$, at $1.8 \mathrm{GPa}$. These result are in good agreement with the reported theoretical and experimental values in the literature..$^{\mathbf{2 0 , 4 5 , 4 6}}$ Therefore, these Raman scattering features may be assigned according to the reports, as shown in Fig. 6 and Table 1. The evolution of these Raman mode frequencies against pressure is shown in Fig. 6(c). All the observed Raman mode frequencies increase monotonously in a linear manner with increasing pressure. The anharmonicity in bonding may have intense influence on the compression behaviors of materials under high pressures. The mode Grüneisen parameter $(\gamma)$ is a powerful tool to describe the changes in the harmonicity of bonding and can be directly measured from a single high pressure experiment. Generally,
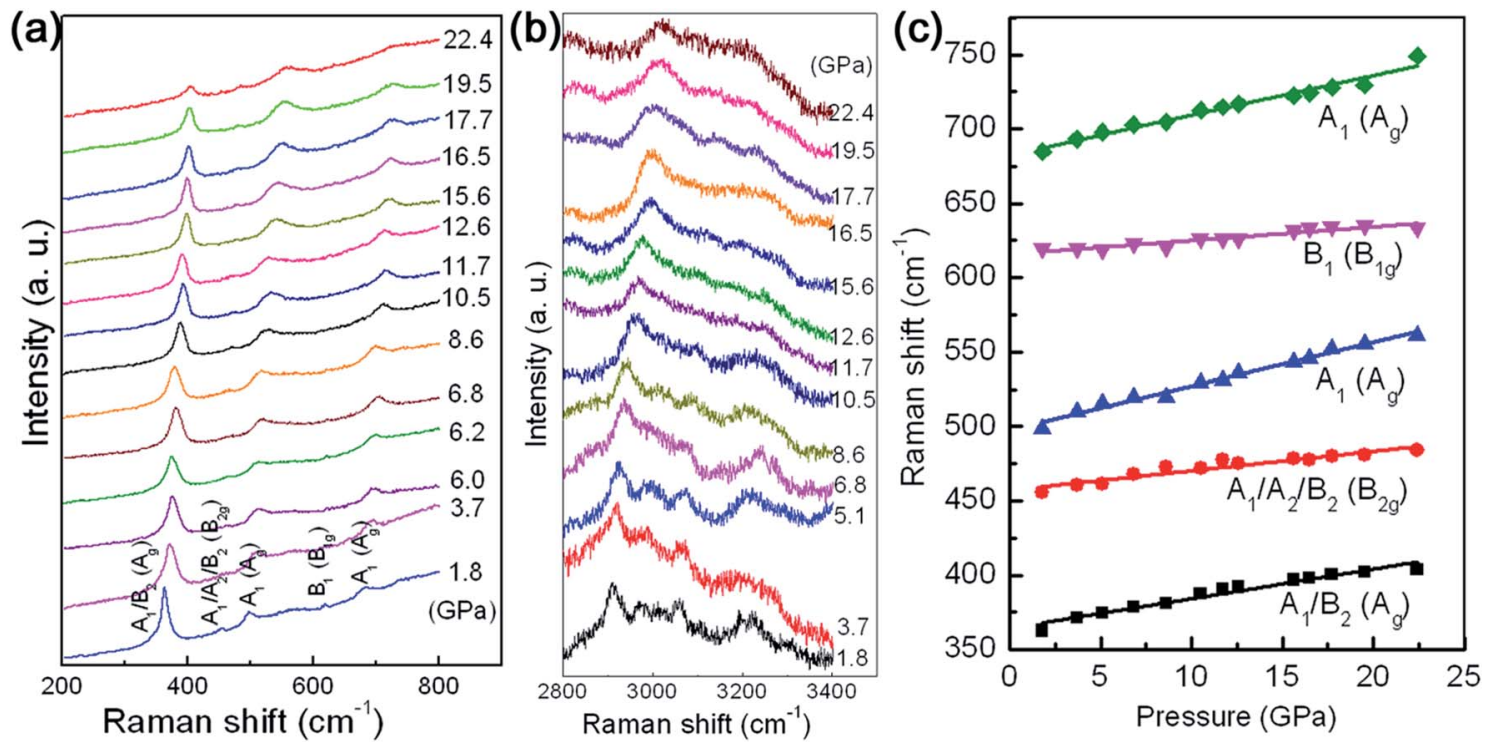

Fig. 6 The selected Raman scattering spectra of $\gamma-\mathrm{AlOOH}$ nanoflakes at high pressures for the modes related with (a) the $\mathrm{Al}-\mathrm{O}$ bonds and (b) the hydrogen bonds. (c) shows the pressure dependences of the Al-O related Raman shifts. 
Table 1 The observed $\mathrm{Al}-\mathrm{O}$ related Raman active modes of $\gamma$ - $\mathrm{AlOOH}$ nanoflakes with their frequencies, assignments and Grüneisen parameters

\begin{tabular}{lllll}
\hline \multirow{2}{*}{\begin{tabular}{l} 
Raman shift $\begin{array}{lll}\text { Assignment } \\
\left(\mathrm{cm}^{-1}\right)\end{array}$ \\
\cline { 2 - 3 }$n$
\end{tabular}} & $C m c 2_{1}$ & $\mathrm{Cmcm}$ & $\begin{array}{l}\mathrm{d} \omega / \mathrm{d} P \\
\left(\mathrm{~cm}^{-1} \mathrm{GPa}^{-1}\right)\end{array}$ & $\begin{array}{l}\text { Grüneisen } \\
\text { coefficient } \gamma\end{array}$ \\
\hline 364 & $\mathrm{~A}_{1} / \mathrm{B}_{2}$ & $\mathrm{~A}_{\mathrm{g}}$ & 1.98 & 0.74 \\
457 & $\mathrm{~A}_{1} / \mathrm{A}_{2} / \mathrm{B}_{2}$ & $\mathrm{~B}_{2 \mathrm{~g}}$ & 1.31 & 0.39 \\
498 & $\mathrm{~A}_{1}$ & $\mathrm{~A}_{\mathrm{g}}$ & 2.94 & 0.80 \\
616 & $\mathrm{~B}_{1}$ & $\mathrm{~B}_{1 \mathrm{~g}}$ & 0.92 & 0.20 \\
683 & $\mathrm{~A}_{1}$ & $\mathrm{~A}_{\mathrm{g}}$ & 2.67 & 0.53
\end{tabular}

the values of mode Grüneisen parameters may be estimated using the following equation:

$$
\gamma=-(\mathrm{d} \ln \omega) /(\mathrm{d} \ln V)=(B / \omega)(\mathrm{d} \omega / \mathrm{d} P)
$$

where $\omega$ is the mode frequency, $V$ is the unit cell volume, $B$ is the bulk modulus, and $P$ is the pressure. The list of all the observed modes along with their frequencies, assignments and Grüneisen parameters are summarized in Table 1. As to the high frequency region, the situation is quite puzzling. Several broad bands may be discerned near 2913, 2971, 3013, 3059, 3191 , and $3226 \mathrm{~cm}^{-1}$ at low pressure (e.g. $\left.1.8 \mathrm{GPa}\right)$. The features at 3059 and $3226 \mathrm{~cm}^{-1}$ have also been reported in the literature and assigned to the stretching modes of $\mathrm{OH}$ groups. ${ }^{\mathbf{4 5 , 4 6}}$ However, any tentative assignments of the rest ones seem uncertain and arbitrary. At elevated pressures, the deteriorating of the signal/noise ratio, together with the broadening and weakening of the bands, makes rational deconvolution of the spectral profiles hardly available. Due to the presence of two interacting $\mathrm{OH}$ groups in the unit cell, six modes may be involved in the vibrations of $\mathrm{OH}$ groups, including two stretching, two in-plane and two out-of-plane bending modes. The peaks corresponding to essentially the same mode and having different symmetry in different space groups may appear at roughly the same frequency. They may be forbidden in one group and not in the others. Thus the experimental spectra in this region may be affected by large uncertainty due to loss of local symmetry. This is not surprising, as actually differences among the correlative space groups are very small, and only involve $\mathrm{H}$ atoms. In addition, the effects of water or other $\mathrm{OH}$ containing molecules adsorbed on the surface cannot be ruled out completely.

\section{Conclusions}

In summary, we synthesized $\gamma$-AlOOH nanocrystals by using a one-step, template-free solvothermal method via an alcoholysis reaction. The structural and compositional characterizations through XRD and EDS analyses showed that the prepared sample consisted of pure orthorhombic $\gamma$-AlOOH with high crystallinity. The morphological studies via SEM and TEM techniques revealed that the prepared sample was comprised of nanometer-scaled single crystalline thin flakes with well- defined shape and high aspect ratio. The optical properties of the obtained $\gamma$-AlOOH nanoflakes are characteristic of an average optical bandgap of $3.35 \mathrm{eV}$, with a broad, asymmetric PL emission band at about $480 \mathrm{~nm}$. In situ high pressure synchrotron radiation ADXRD studies indicated a strong anisotropy of the linear compressibilities of $\gamma$-AlOOH nanoflakes, with the $b$ axis being much more compressible than the $a$ and $c$ axes. Fitting the pressure-volume data to the third-order Birch-Murnaghan equation of state yielded the bulk modulus of $B_{0}=135.2 \mathrm{GPa}$, higher than the theoretical value of bulk $\gamma$ AlOOH. Such reduced compressibility was suggested to be related with the unique intrinsic geometry in the nanoflakes. By following the evolution of the Raman shifts against pressure, the Grüneisen parameters of the observed Raman modes related with the intralayer $\mathrm{Al}-\mathrm{O}$ bonds were estimated, which may have important implications on the mechanism of the anisotropic linear compression behaviors of $\gamma-\mathrm{AlOOH}$ nanoflakes.

\section{Acknowledgements}

This work is supported partially by the National Natural Science Foundation of China (Grant no. 11304114). High pressure experiments were carried out at $4 \mathrm{~W} 2$ beamline, Beijing Synchrotron Radiation Facility (BSRF).

\section{Notes and references}

1 Y. Jun, J. Choi and J. Cheon, Angew. Chem., Int. Ed., 2006, 45, 3414-3439.

2 M. Huang and P. Lin, Adv. Funct. Mater., 2012, 22, 14-24.

3 B. Sun, Z. Ji, Y. Liao, M. Wang, X. Wang, J. Dong, C. Chang, R. Li, H. Zhang, A. Nel and T. Xia, ACS Nano, 2013, 7, 1083210849.

4 S. Liu, C. Chen, Q. Liu, Y. Zhuo, D. Yuan, Z. Dai and J. Bao, RSC Adv., 2015, 5, 71728.

5 J. Zhang, S. Liu, J. Lin, H. Song, J. Luo, E. Elssfah, E. Ammar, Y. Huang, X. Ding, J. Gao, S. Qi and C. Tang, J. Phys. Chem. B, 2006, 110, 14249-14252.

6 P. Pardo, N. Montoya and J. Alarcón, CrystEngComm, 2015, 17, 2091.

7 J. Wen, M. Liu and C. Mou, CrystEngComm, 2015, 17, 1959.

8 X. Song, P. Yang, C. Jia, L. Chen and K. Matras-Postolek, RSC Adv., 2015, 5, 33155.

9 X. Liu, C. Niu, X. Zhen, J. Wang and X. Su, J. Colloid Interface Sci., 2015, 452, 116-125.

10 G. Liu, H. Ye, Y. Dai, L. Yu, H. Jiang, Y. Zhou and K. Han, J. Alloys Compd., 2015, 647, 18-23.

11 G. Ji, M. Li, G. Li, G. Gao, H. Zou, S. Gan and X. Xu, Powder Technol., 2012, 215-216, 54-58.

12 Y. Zhang, Y. Jia, Z. Jin, X. Yu, W. Xu, T. Luo, B. Zhu, J. Liu and X. Huang, CrystEngComm, 2012, 14, 3005-3007.

13 X. Duan, T. Kim, D. Li, J. Ma and W. Zheng, Chem.-Eur. J., 2013, 19, 5924-5937.

14 Z. Tang, J. Liang, X. Li, J. Li, H. Guo, Y. Liu and C. Liu, J. Solid State Chem., 2013, 20, 305-314. 
15 Y. Xia, X. Jiao, Y. Liu, D. Chen, L. Zhang and Z. Qin, J. Phys. Chem. C, 2013, 117, 15279-15286.

$16 \mathrm{Z} . \mathrm{Xu}, \mathrm{J}$. Yu, J. Low and M. Jaroniec, ACS Appl. Mater. Interfaces, 2014, 6, 2111-2117.

17 C. Corbató, R. Tettenhorst and G. Christoph, Clays Clay Miner., 1985, 33, 71-75.

18 X. Bokhimi, J. Toledo-Antonio, M. Guzmán-Castillo and F. Hernández-Beltrán, J. Solid State Chem., 2001, 159, 32-40.

19 D. Chiche, M. Digne, R. Revel, C. Chanéac and J. P. Jolivet, J. Phys. Chem. C, 2008, 112, 8524-8533.

20 Y. Noel, R. Demichelis, F. Pascale, P. Ugliengo, R. Orlando and R. Dovesi, Phys. Chem. Miner., 2009, 36, 47-59.

21 A. Suzuki, E. Ohtani and T. Kamada, Phys. Chem. Miner., 2000, 27, 689-693.

22 E. Ohtani, K. Litasov, A. Suzuki and T. Kondo, Geophys. Res. Lett., 2001, 28, 3991-3993.

23 A. Sano, E. Ohtani, T. Kubo and K. Funakoshi, J. Phys. Chem. Solids, 2004, 65, 1547-1554.

24 H. Mao, J. Shu, J. Hu and R. Hemley, Solid State Commun., 1994, 90, 497-500.

25 J. Xu, J. Hu, L. Ming, E. Huang and H. Xie, Geophys. Res. Lett., 1994, 21, 161-164.

26 M. Schmidt, Am. Mineral., 1995, 80, 1286-1292.

27 K. Grevel, M. Burchard, D. Fasshauer and T. Peun, J. Geophys. Res.: Solid Earth, 2000, 105, 27877-27887.

28 B. Winkler, M. Hytha, C. Pickard, V. Milman, M. Warren and M. Segall, Eur. J. Mineral., 2001, 13, 343-349.

29 A. Friedrich, D. Wilson, E. Haussühl, B. Winkler, W. Morgenroth, K. Refson and V. Milman, Phys. Chem. Miner., 2007, 34, 145-157.

30 A. Friedrich, E. Haussühl, R. Boehler, W. Morgenroth, E. Juarez-Arellano and B. Winkler, Am. Mineral., 2007, 92, 1640-1644.
31 A. Sano, E. Ohtani, T. Kondo, N. Hirao, T. Sakai, N. Sata, Y. Ohishi and T. Kikegawa, Geophys. Res. Lett., 2008, 35, L03303.

32 A. Sano-Furukawa, H. Kagi, T. Nagai, S. Nakano, S. Fukura, D. Ushijima, R. Iizuka, E. Ohtani and T. Yagi, Am. Mineral., 2009, 94, 1255-1261.

33 T. Kuribayashi, A. Sano-Furukawa and T. Nagase, Phys. Chem. Miner., 2014, 41, 303-312.

34 D. Tunega, H. Pašalić, M. Gerzabek and H. Lischka, J. Phys.: Condens. Matter, 2011, 23, 404201.

35 A. Hammersley, S. Svensson, M. Hanfland, A. Fitch and D. Hausermann, High Pressure Res., 1996, 14, 235-248.

36 B. Toby, J. Appl. Crystallogr., 2001, 34, 210-213.

37 A. Alemi, Z. Hosseinpour, M. Dolatyari and A. Bakhtiari, Phys. Status Solidi B, 2012, 249, 1264-1270.

38 Z. Yu, C. Wang, X. Gu and C. Li, J. Lumin., 2004, 106, 153157.

39 P. Gao, Y. Xie, Y. Chen, L. Ye and Q. Guo, J. Cryst. Growth, 2005, 285, 555-560.

40 B. Chen, D. Penwell and M. B. Kruger, Solid State Commun., 2000, 115, 191-194.

41 C. Grant, J. Crowhurst, T. Arsenlis, E. Bringa, Y. Wang, J. Hawreliak, P. Pauzauskie and S. Clark, J. Appl. Phys., 2009, 105, 084311.

42 D. Ragan, D. Clarke and D. Schiferl, Rev. Sci. Instrum., 1996, 67, 494.

43 Z. Wang, S. Saxena, V. Pischedda, H. Liermann and C. Zha, Phys. Rev. B: Condens. Matter, 2001, 64, 012102.

44 Z. Wang, Y. Zhao, D. Schiferl, J. Qian, R. Downs, H. Mao and T. Sekine, J. Phys. Chem. B, 2003, 107, 14151.

45 A. Kiss, G. Keresztury and L. Farkas, Spectrochim. Acta, Part A, 1980, 36, 653-658.

46 H. Ruan, R. Frost and J. Kloprogge, J. Raman Spectrosc., 2001, 32, 745-750. 